\title{
Taming ROS: Mitochondria-Targeted AlEgen for Neuron Protection via Photosensitization-Triggered Autophagy
}

\author{
Chao Chen, ${ }^{\dagger, \#}$ Ruoyao Zhang, ${ }^{\dagger, \#}$ Jianyu Zhang, ${ }^{\dagger, \#}$ Yufan Zhang, ${ }^{\ddagger}$ Haoke Zhang, ${ }^{\ddagger}$ Zaiyu \\ Wang, ${ }^{\dagger}$ Xiaolin Huang, ${ }^{\dagger}$ Sijie Chen, ${ }^{\xi}$ Ryan T. K. Kwok, ${ }^{\dagger}$ Jacky W. Y. Lam, ${ }^{\dagger}$ Dan Ding ${ }^{*, \neq, \S}$ \\ and Ben Zhong Tang*,t,s,s,L
}

${ }^{\dagger}$ Department of Chemistry, Hong Kong Branch of Chinese National Engineering Research Center for Tissue Restoration and Reconstruction, Institute for Advanced Study, State Key Laboratory of Molecular Nanoscience, Division of Life Science, and Department of Chemical and Biological Engineering, The Hong Kong University of Science and Technology, Clear Water Bay, Kowloon, Hong Kong 999077, China.

¡State Key Laboratory of Medicinal Chemical Biology, Key Laboratory of Bioactive Materials, Ministry of Education, and College of Life Sciences, Nankai University, Tianjin 300071, China

sMing Wai Lau Centre for Reparative Medicine, Karolinska Institutet, Hong Kong

${ }^{\S}$ Center for Aggregation-Induced Emission, SCUT-HKUST Joint Research Institute, State Key

Laboratory of Luminescent Materials and Devices, South China University of Technology, Guangzhou 510640, China

${ }^{\perp}$ AIE Institute, Guangzhou Development District, Huangpu, Guangzhou 510530, China

KEYWORDS. Controlling ROS generation, mitochondria-targeted AlEgen, neuron protection, autophagy

\begin{abstract}
Oxidative damages lead to accumulated harmful wastes, which in turn aggravate the related diseases and ROS imbalance. Therefore, provoking the defense system against severe oxidation and maintaining ROS homeostasis are desired. Herein, we used a mitochondria-targeted aggregationinduced emission luminogen (AIEgen) as a phototherapy agent for neuron protection by virtue of its efficient ROS generation in aggregates and mitochondrial delivery. It is demonstrated that controllable ROS generation within mitochondria can trigger defensive autophagy against oxidative damages in neuron cells. This work not only verifies the concept that taming ROS can be used for cell protection, but also provides a promising method to trigger autophagy against destructive oxidation, displaying broad prospects for alleviating oxidation-related diseases and promoting cell-based therapy.
\end{abstract}

Life is a contradictory organism full of dynamic balance, such as immune homeostasis, energy equilibrium, reactive oxygen species (ROS) balance, etc. ${ }^{1-3}$ The balance of these dynamic systems is finely tuned in a normal range to keep fit. Among various dynamic systems, ROS balance has drawn great attention due to its critical role and extensive participation in life and disease process..$^{3-5}$ For example, ROS with normal level is closely involved in cell differentiation and development, whereas too much ROS will lead to superfluous oxidized substances, which in turn exacerbate the ROS imbalance and elicit many severe diseases (e.g., cancer, inflammation, neurodegenerative diseases and ischemia- reperfusion injury)..$^{4-6}$ Thereby, developing agents or methods to maintain the ROS homeostasis and timely clean the harmful wastes is highly desired.

At present, antioxidants (such as N-Acety-LCysteine (NAC), Vitamin C, Vitamin E, etc.) are commonly used to maintain the ROS balance. ${ }^{7}$ Although antioxidants can quickly scavenge redundant ROS, they can neither remove the accumulated harmful wastes nor generate durable protection. In contrast, autophagy inducers (e.g., rapamycin, resveratrol and many natural compounds) can improve the level of autophagy, which enables cell to digest harmful substances via cell's own defense system to achieve a long-term protection. ${ }^{8}$ However, most 
autophagy inducers belong to chemotherapy agents, and the lack of specific and controllable delivery and visual tracking contribute to some common drawbacks, such as multiple targets, organ toxicity, and elusive mechanism, 9-11 limiting their further application.

Compared to chemotherapy, phototherapy owns inherent advantages of high selectivity and super temporal-spatial resolution, exhibiting promising prospect for specific delivery and accurate treatment. ${ }^{12-17}$ Photodynamic therapy (PDT) is a successful demonstration of phototherapy in precise treatment of diseases and is easy to achieve localized delivery of massive ROS via engineering control of light. ${ }^{18-22}$ Inspired by this, we wonder whether phototherapy can be used to trigger cell's own protective system to remove harmful oxidized components and maintain the ROS balance. According to literatures, moderate ROS derived from mitochondria is crucial for stimulating the cell's defense system, especially for autophagy to remove harmful wastes. ${ }^{23-24}$ Thereby, provoking cell's defensive autophagy via photo-controlled ROS generation within mitochondria was proposed.

Traditional photosensitizers (PS) usually possess conjugated planar structures and they are easy to form aggregates in physiological environment. After excitation, these PS will predominantly consume energy through heat production due to strong intermolecular $\pi-\pi$ interactions in the aggregate state, leading to reduced ROS generation as well as fluorescence emission. Therefore, it's hard to accurately and effectively control ROS generation. ${ }^{25}$ Contrary to traditional PS, AlEgenbased photosensitizers (AIE-PS) are able to effectively produce ROS and fluorescence in aggregates thanks to the twisted structures, which strongly inhibits the thermal decay. ${ }^{26-30}$ Thus, we can conveniently control ROS at different amount and exact location via AIE-PS based phototherapy.

Based on these considerations, two mitochondria-targeted AIE-PS (DTCSPY and DTCSPE) with twisted structures were designed. As both intermolecular interactions (e.g., $\pi-\pi$ interaction) and intramolecular motions are major pathway to consume energy by heat generation, ${ }^{31-32}$ comparatively rigid alkyne group was introduced into the structure of DTCSPY to further hinder thermal dissipation through intramolecular motions. For comparison, a more flexible alkene-based $\pi$ bridge was introduced into DTCSPE. As expected, the ROS generation efficiency of DTCSPY is much better than that of DTCSPE both in PBS solutions and live cells. Particularly, taming DICSPY-generated ROS for cell protection against severe oxidation via photosensitization-triggered autophagy was confirmed. The experimental results also showed that AIE-PS exhibited a better protective effect than the widely used antioxidants (NAC and Vitamin C).

DTCSPY and DTCSPE were synthesized through Knoevenagel condensation and methylation as shown in Figure $1 \mathrm{~A}$ and Scheme S1. The chemical structures of the two molecules were fully characterized by ${ }^{1} \mathrm{H} N M R$, ${ }^{13} \mathrm{C}$ NMR, and HRMS. DTCSPY and DTCSPE share the same electron donating and accepting skeleton but different $\pi$-bridges. Pyridinium was introduced as mitochondria-targeted moiety. Then photophysical properties of DTCSPY and DTCSPE were investigated. As shown in Figure $1 \mathrm{~B}$ and Figure S2, the absorption maxima of DTCSPY and DTCSPE were $490 \mathrm{~nm}$, and the emission spectra were centered at $700 \mathrm{~nm}$ and $710 \mathrm{~nm}$ of DTCSPY and DTCSPE in DMSO/water mixtures, respectively. Figure $1 C$ and $D$ showed that both of them exhibited typical AIE activity and remarkable ROS generation capability much better than rose bengal (a well-known PS) under the same condition. Furthermore, DTCSPY decorated with rigid alkyne-based $\pi$ bridge displayed better performance in both AIE activity and ROS production capability than that of DTCSPE featured with flexible alkenebased $\pi$-bridge (Figure 1C, D, and S3). These results indicated that rigid alkyne in AIEgens could further improve ROS generation ability by hindering thermal dissipation from intramolecular motions.

Theoretical calculation was conducted to understand the relationship between chemical structures and different ROS generation performance by density functional theory (DFT) at the level of CAM-B3LYP/6-31G(d,p). ${ }^{33}$ Optimized geometries and electron cloud distribution separately depicted comparable large dihedral angle of stilbene segment $(>$ $21^{\circ}$ ) and relatively distant HOMO (highest occupied molecular orbital)-LUMO (lowest unoccupied molecular orbital) separation in both AIE-PS (Figure 2A), which contributed to twisted structures, AIE activity and efficient ROS generation in aggregates. To further explain the different ROS performance of DTCSPY and DTCSPE, the energy gap $(\Delta E)$ and spin-orbital coupling (SOC) between singlet and triplet states were analyzed. ${ }^{34} \mathrm{Although}$ the results showed almost the same level of SOC efficiency of the two compounds, the $\Delta E$ between S1 and T2 state of DTCSPY is much smaller than that of DTCSPE, which is mainly responsible for its higher ROS generation efficiency (Figure 2B). 

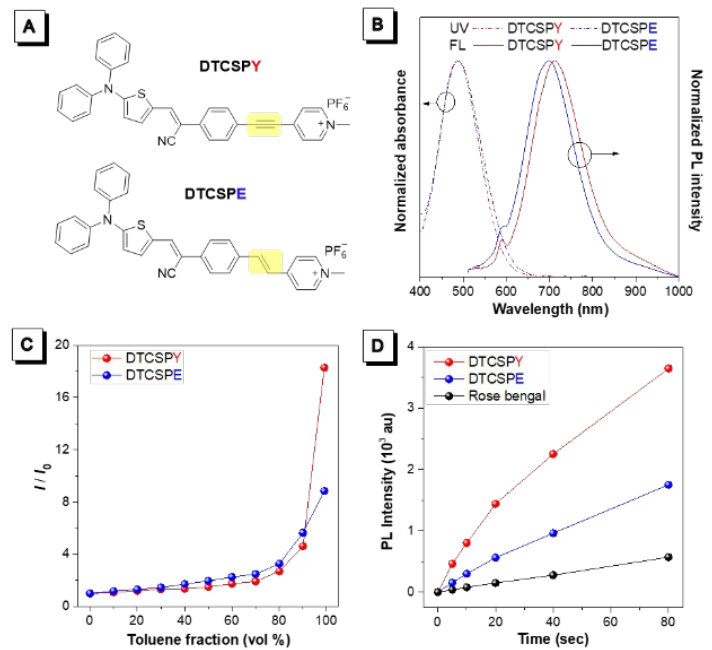

Figure 1. (A) Chemical structures of DTCSPY and DTCSPE. (B) Normalized UV/PL spectra of DTCSPY and DTCSPE in DMSO/water (1:99) mixtures. (C) Plots of relative emission intensity $(I / / 0)$ of DTCSPY and DTCSPE in mixtures of DMSO/toluene with different toluene fractions (vol \%). (D) Plots of fluorescence intensity of DCF at $520 \mathrm{~nm}$ in PBS involving different photosensitizers (5 $\mu \mathrm{M})$ : DTCSPE, DTCSPY and rose Bengal versus different irradiation times, respectively

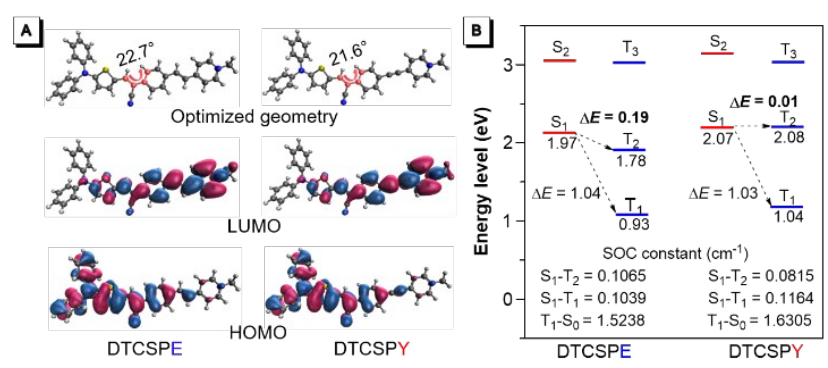

Figure 2. (A) Optimized geometries and electron cloud distributions of HOMO and LUMO of DTCSPE and DTCSPY. (B) Diagram of energy level of singlet and triplet state of DTCSPE and DTCSPY based on optimized singlet geometries. $\Delta E$ and SOC constants are also indicated.

As many serious neuron diseases are closely associated with ROS imbalance, oxidative damages and overloaded oxidized wastes, ${ }^{35-36}$ we used HT22 murine hippocampal neuronal cells as a promising demonstration to validate the concept that taming ROS generation can trigger defense against the severely oxidative damages. First, mitochondria-targeted ability of DTCSPY and DTCSPE were evaluated in HT22 neuron cells through co-staining with commercial mitochondrial probe MitoTracker Deep Red FM (MTDR). As depicted in Figure 3A$C$ and Figure S4, both DTCSPY and DTCSPE can illuminate the mitochondria with high signal to background ratio, owing to excellent mitochondria-targeted ability and AIE activity. Pearson's correlation coefficient between DTCSPY/DTCSPE and MTDR both reached $\sim 0.86$, suggesting outstanding mitochondriatargeted capability. Subsequently, ROS generation efficiency of DTCSPY and DTCSPE in
HT22 neuron cells were investigated using $2^{\prime}, 7^{\prime}$ dichlorodihydrofluorescein diacetate (DCF-DA). From the results, HT22 cells themselves cannot oxidize the DCF-DA to highly green-emissive DCF (Figure 3D), and the cells without light irradiation (Figure 3E) or AIE-PS (Figure 3F) can also hardly be collected DCF fluoresce signals. In contrast, after treatment with AIE-PS (DTCSPY and DTCSPE) and light irradiation, significant signals could be observed, indicating that efficient ROS was generated in HT22 neuron cells (Figure 3G-H). Figure 31 showed that NAC (a ROS scavenger) could suppress the strong signals, further demonstrating that it was indeed the ROS generated by AIE-PS that oxidized DCF-DA to DCF. Additionally, compared with DTCSPE-treated group, DTCSPY-treated cells exhibited much stronger fluorescence, displaying more efficient ROS generation, which was consistent with the results of theoretical calculation and in vitro tests in PBS solutions.
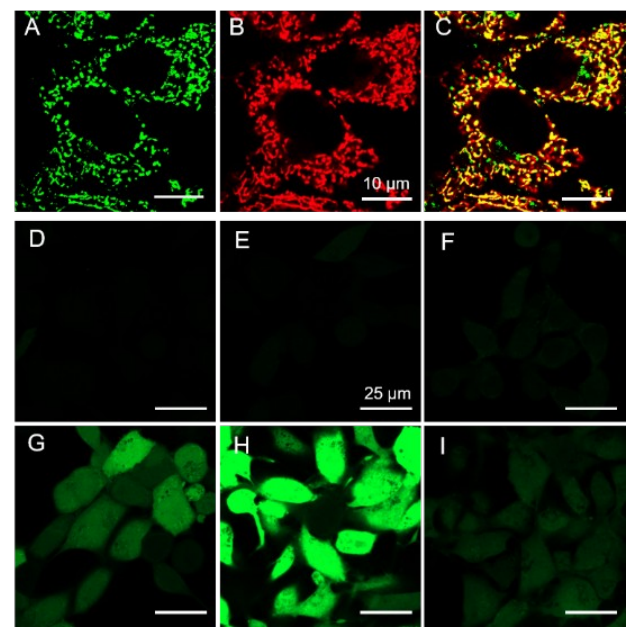

Figure 3. (A-C) Confocal laser scanning microscopy (CLSM) images of colocalization between $5 \mu \mathrm{M}$ DTCSPY (A) and $1 \mu \mathrm{M}$ MTDR (B) in HT22 neuronal cells; (C) Overlap between (A) and (B); Scale bar: $10 \mu \mathrm{m}$. (D-I) CLSM images indicate ROS production in HT22 neuronal cells treated with pure DCF-DA (D), "DTCSPY + DCF-DA" (E), "light + DCF-DA" (F), "DTCSPE + DCF-DA + light" (G), DTCSPY + DCF-DA + light" $(H)$, and "DTCSPY + DCF-DA + light + NAC" (I). Green fluorescence: DCF. DTCSPE/DTCSPY: $5 \mu$; DCF-DA: $25 \mu \mathrm{M}$; Scale bar: $25 \mu \mathrm{m}$.

After verifying the mitochondria-targeted and ROS generation capabilities of AIE-PS in HT22 neuron cells, we examined whether controllable ROS generation within mitochondria can trigger a defensive autophagy against oxidative damage. Firstly, we confirmed that both DTCSPY and DTCSPE owned good biocompatibility in darkness by MTT [3-(4,5dimethylthiazol-2-yl)-2,5-diphenyltetrazolium bromide] assay (Figure 4A). Then, we optimized experimental conditions to control the amount of the generated ROS via treating the cells with AIE-PS in different concentrations under light irradiation. As displayed in Figure 4B, when incubated concentrations of DTCSPY and 
DTCSPE are lower than or equal to $0.5 \mu \mathrm{M}$ and $2.5 \mu \mathrm{M}$, respectively, the cell viability of HT22 neuron cells was higher than $85 \%$, indicating that the amount of ROS generated within these ranges (0-0.5 $\mu \mathrm{M}$ for DTCSPY and 0-2.5 $\mu \mathrm{M}$ for DTCSPE) was tolerable in neuron cells. Next, for comparison, the concentration of $0.5 \mu \mathrm{M}$ was selected for DTCSPY and DTCSPE to explore the protective effect by adjusting the irradiation time. Briefly, HT22 neuron cells were incubated with $0.5 \mu \mathrm{M}$ DTCSPY and DTCSPE for $90 \mathrm{~min}$, then treated with light irradiation for different time $(0,1,3$, and $5 \mathrm{~min})$. After further $6 \mathrm{~h}$ incubation, the cells were subsequently exposed to $100 \mu \mathrm{M}$ of hydrogen peroxide $\left(\mathrm{H}_{2} \mathrm{O}_{2}\right)$ for $1 \mathrm{~h}$ and finally measured at $48 \mathrm{~h}$. As shown in Figure 4C, the protective effect of neuron cells increased with prolonged irradiation time, suggesting that the amount of ROS generated by AIE-PS needs to reach a certain level to trigger cell's defense system. Compared with DTCSPE-treated cells, DTCSPY-treated cells exhibited much stronger protective effect as DTCSPY was easier to reach the ROS threshold of provoking defense system owing to its more efficient ROS generation upon light irradiation. Moreover, bright field pictures and CLSM images also described the differences of healthy state and quantity of live or dead cells between DTCSPY-treated group and control group, respectively (Figure S5), which were consistent with the protective effect in Figure 4C. Finally, both NAC and Vitamin C, the widely used agents for resisting oxidative damages, were selected for comparison. As shown in Figure 4D, DTCSPY can trigger a more effective protection against $\left(\mathrm{H}_{2} \mathrm{O}_{2}\right)$ than NAC and Vitamin $\mathrm{C}$, demonstrating the importance of long-term defense triggered by DTCSPY. In addition, in CQ (chloroquine, an autophagy inhibitor)-added group, the protective effect of DTCSPY treatment was lost, suggesting this protection was mediated by autophagy.

Further experiments were conducted to confirm that the protective effect was definitely from DTCSPY-triggered autophagy at this optimized condition. As the punctate distribution of LC3B is one of the best hallmarks of autophagy induction, immunofluorescence staining of LC3B were analyzed using CLSM. ${ }^{37}$ As shown in Figure 5A, "Control" group without any treatment and "DTCSPY" group without light irradiation exhibited diffused distribution of LC3B, while massive LC3B puncta were observed in "DTCSPY + Light" group at $6 \mathrm{~h}$ after irradiation, which indicated that effective autophagy was induced by DTCSPY-generated ROS. Quantified results also revealed a 6.4-fold and a 5.0-fold increase of LC3B puncta in “DTCSPY + Light" group
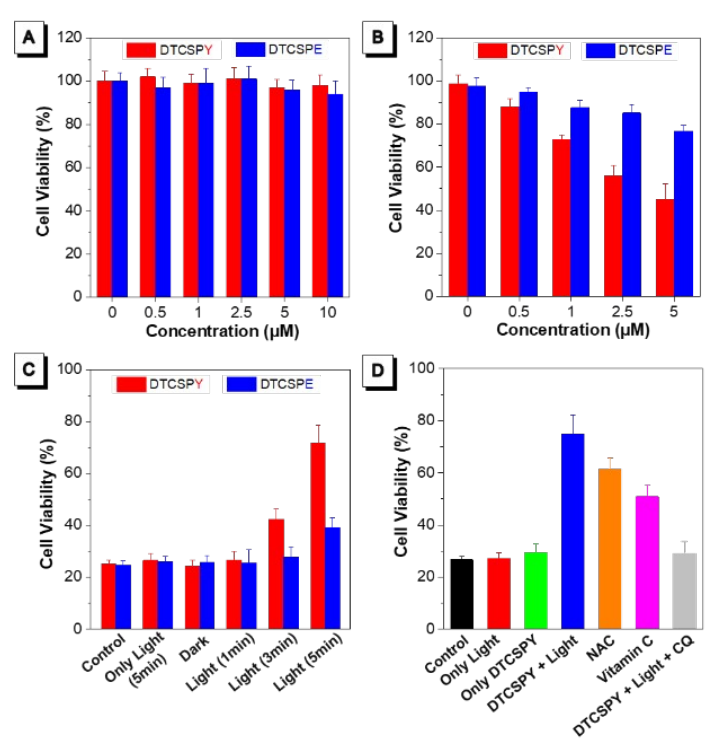

Figure 4. (A) Viability of HT22 neuronal cells treated with different concentrations of DTCSPY and DTCSPE in darkness for $24 \mathrm{~h}$. (B) Viability of HT22 neuronal cells incubated with different concentrations of DTCSPY and DTCSPE, under light irradiation $\left(50 \mathrm{~mW} / \mathrm{cm}^{2}\right)$ for $5 \mathrm{~min}$ and measured at $24 \mathrm{~h}$. (C) Evaluation of the protective effect against $\mathrm{H}_{2} \mathrm{O}_{2}(100 \mu \mathrm{M})$ in $\mathrm{HT} 22$ neuronal cells treated with $0.5 \mu \mathrm{M}$ DTCSPY and DTCSPE at $48 \mathrm{~h}$, respectively. (D) Determination of protective effect against $\mathrm{H}_{2} \mathrm{O}_{2}$ in $\mathrm{HT} 22$ cells treated with different agents at $48 \mathrm{~h}$. Light irradiation: $50 \mathrm{~mW} / \mathrm{cm}^{2}$. DTCSPY/DTCSPE: $0.5 \mu \mathrm{M}$. Vitamin C: $100 \mu \mathrm{M}$. NAC: $10 \mathrm{mM}$. CQ: $50 \mu \mathrm{M} . \mathrm{H}_{2} \mathrm{O}_{2}$-treated group served as control group. All cell viability values were divided by the group without any treatment for comparison convenience.

than that of "Control" and "DTCSPY" groups. Next, the LC3B conversion from full-length LC3B-I to LC3B-II was detected at $6 \mathrm{~h}$ by western blots, as it is another important hallmark of autophagy. As depicted in Figure 5B and S6, the ratio of LC3B-II / LC3B-I in "DTCSPY + Light" group was 1.8- fold higher than that of "Control" and "DTCSPY" group, suggesting activated autophagy in experimental group. Moreover, two other necessary proteins (ATG5 and Beclin 1) involved in autophagy were also elevated in "DTCSPY + Light" group compared with the other two groups. ${ }^{38}$ These results together verified that controlling the ROS generation within mitochondria using DTCSPY can effectively trigger autophagy, which contributed to the protective effect of neuron cells from severely oxidative damage.

In summary, two highly effective and mitochondria-targeted AIE-PS (DTCSPY and DTCSPE) were designed and synthesized. DTCSPY constructed with alkyne-containing $\pi$ bridge, exhibited better performance than DTCSPE featured with alkane-containing $\pi$ bridge in AIE activity and ROS generation efficiency due to less thermal dissipation in relatively rigid skeleton. HT22 neuron cells were used as a model to demonstrate the concept 
that taming ROS generation can trigger cell protection against severely oxidative damages. To the best of our knowledge, this demonstration is the first attempt to trigger neuron protection by control of ROS via phototherapy.

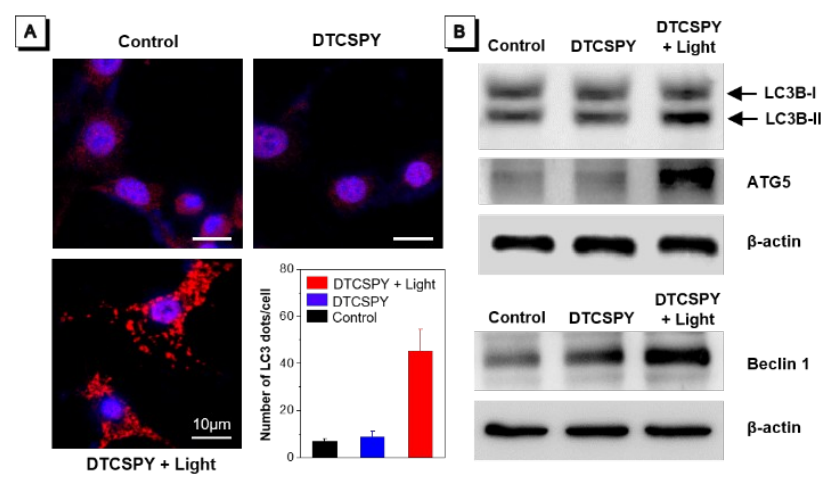

Figure 5. (A) Representative CLSM images and quantitative analysis of LC3B dots in different groups. LC3B (red signals) indicates autophagosome. Scale bar: $10 \mu \mathrm{m}$. (B) Mechanism analysis of cytoprotective autophagy with different treatment using western blots.

Besides, we have applied this method to protect mesenchymal stem cells from oxidation-induced aging and death. Not only does it provide a useful strategy against oxidative damages caused by acute inflammation, neurodegenerative diseases, ischemia-reperfusion injury, serious side-effect of radiotherapy, and chemotherapy, but also exhibits great potential to improve cell survival in oxidative environment during stem-cell transplantation and chimeric antigen receptor $\mathrm{T}$ (CAR-T)-cell based therapy. ${ }^{39-40}$

\section{ASSOCIATED CONTENT}

\section{Supporting Information.}

The Supporting Information is available free of charge on the ACS Publications website at DOI.

Experimental procedures, chemical synthesis and characterization of compounds, and Supplementary figures.

\section{AUTHOR INFORMATION}

\section{Corresponding Author}

* dingd@nankai.edu.cn

* tangbenz@ust.hk

\section{Author Contributions}

${ }^{\#}$ C.C., R.Z. and J.Z contributed equally to this work.

\section{Notes}

The authors declare no competing financial interest.

\section{ACKNOWLEDGMENT}

This work was financially supported by the National Science Foundation of China (21788102, 51873092, and 51961160730), the Research
Grants Council of Hong Kong (16305518, 16305618, N-HKUST609/19, A-HKUST605/16 and C6009-17G), the Innovation and Technology Commission (ITC-CNERC14SC01 and ITCPD/17-9), Ming Wai Lau Centre for Reparative Medicine Associate Member Programme (MWLC19SC02), and the National Key Research and Development Program of China (2017YFE0132200).

\section{REFERENCES}

(1) Honda, K.; Littman, D. R. Nature 2016, 535 , 75-84.

(2) López, M.; Varela, L.; Vázquez, M. J.;

Rodríguez-Cuenca, S.; González, C. R.; Velagapudi, V. R.; Morgan, D. A.; Schoenmakers, E.;

Agassandian, K.; Lage, R. Nat. Med. 2010, 16, 1001-1008.

(3) D'Autréaux, B.; Toledano, M. B. Nat. Rev. Mol. Cell Biol. 2007, 8, 813-824.

(4) Kawagishi, H.; Finkel, T. Nat. Med. 2014, 20, 711-713.

(5) Schieber, M.; Chandel, N. S. Curr. Biol. 2014, 24, R453-R462.

(6) Reczek, C. R.; Chandel, N. S. Curr. Opin. Cell Biol. 2015, 33, 8-13.

(7) Blokhina, O.; Virolainen, E.; Fagerstedt, K. V. Annals of botany 2003, 91, 179-194.

(8) Levine, B.; Packer, M.; Codogno, P. J. Clin. Investig. 2015, 125, 14-24.

(9) Partridge, A. H.; Burstein, H. J.; Winer, E. P. JNCl Monographs 2001, 2001, 135-142.

(10) Wu, X.; Sun, X.; Guo, Z.; Tang, J.; Shen, Y.; James, T. D.; Tian, H.; Zhu, W. J. Am. Chem. Soc. 2014, 136, 3579-3588.

(11) Li, S.; Zhang, Y.; Ho, S.-H.; Li, B.; Wang, M.; Deng, X.; Yang, N.; Liu, G.; Lu, Z.; Xu, J.; Shi,Q.; Han, J. Y.; Zhang, L.; Wu, Y.; Zhao, Y.; Nie, G. Nat. Biomed. Eng. 2020, 4, 732-742.

(12) Vreman, H. J.; Wong, R. J.; Stevenson, D. K. Semin. Perinatol. 2004, 28, 326-333.

(13) Lyu, Y.; Xie, C.; Chechetka, S. A.; Miyako, E.; $\mathrm{Pu}, \mathrm{K}$. J. Am. Chem. Soc. 2016, 138, 9049-9052.

(14) Li, J.; Pu, K. Chem. Soc. Rev. 2019, 48, 3871.

(15) Zhao, X.; Tapec-Dytioco, R.; Tan, W. J. Am. Chem. Soc. 2003, 125, 11474-11475.

(16) Ying, Z.-M.; Wu, Z.; Tu, B.; Tan, W.; Jiang, J.-H. J. Am. Chem. Soc. 2017, 139, 9779-9782.

(17) Li, H.; Yao, Q.; Sun, W.; Shao, K.; Lu, Y.; Chung, J.; Kim, D.; Fan, J.; Long, S.; Du, J.; Li, Y.; Wang, J.; Yoon, J.; Peng, X. J. Am. Chem. Soc. 2020, 142, 6381-6389.

(18) Li, M.; Xia, J.; Tian, R.; Wang, J.; Fan, J.; Du, J.; Long, S.; Song, X.; Foley, J. W.; Peng, X. J. Am. Chem. Soc. 2018, 140, 14851-14859.

(19) Xu, F.; Li, H.; Yao, Q.; Ge, H.; Fan, J.; Sun, W.; Wang, J.; Peng, X. Chem. Sci. 2019, 10, 1058610594.

(20) Li, M.; Shao, Y.; Kim, J. H.; Pu, Z.; Zhao, X.; Huang, H.; Xiong, T.; Kang, Y.; Li, G.; Shao, K.; Fan, J.; Foley, J. W.; Kim, J. S.; Peng, X. J. Am. Chem. Soc. 2020, 142, 5380-5388.

(21) Xu, S.; Yuan, Y.; Cai, X.; Zhang, C.-J.; Hu, F.; Liang, J.; Zhang, G.; Zhang, D.; Liu, B., Chem. Sci. 2015, 6, 5824-5830. 
(22) Mao, D.; Hu, F.; Yi, Z.; Kenry, K.; Xu, S.; Yan, S.; Luo, Z.; Wu, W.; Wang, Z.; Kong, D.; Liu, X.; Liu, Bin. Sci. Adv. 2020, 6, eabb2712.

(23) Scherz-Shouval, R.; Elazar, Z. Trends Cell Biol. 2007, 17, 422-427.

(24) Kim, S.-H.; Kim, K.-Y.; Park, S.-G.; Yu, S.-N.; Kim, Y.-W.; Nam, H.-W.; An, H.-H.; Kim, Y.-W.; Ahn, S.-C., Oncotarget 2017, 8, 111581.

(25) Lovell, J. F.; Liu, T. W.; Chen, J.; Zheng, G. Chem. Rev. 2010, 110, 2839-2857.

(26) Chen, C.; Ni, X.; Jia, S.; Liang, Y.; Wu, X.; Kong, D.; Ding, D. Adv. Mater. 2019, 31, 1904914 (27) Hu, F.; Xu, S.; Liu, B., Adv. Mater. 2018, 30 , 1801350.

(28) Yu, C. Y.; Xu, H.; Ji, S.; Kwok, R. T.; Lam, J. W.; Li, X.; Krishnan, S.; Ding, D.; Tang, B. Z. Adv. Mater. 2017, 29, 1606167.

(29) TungáLeung, C. W.; YipáLam, J. W.; ZhongáTang, B. Chem. Commun. 2014, 50, 14451-14454.

(30) Chen, C.; Ou, H.; Liu, R.; Ding, D. Adv. Mater. 2020, 32, 1806331.

(31) Chen, C.; Ni, X.; Jia, S.; Liang, Y.; Wu, X.; Kong, D.; Ding, D. Adv. Mater. 2019, 31, 1904914. (32) Zhao, Z.; Chen, C.; Wu, W.; Wang, F.; Du, L.; Zhang, X.; Xiong, Y.; He, X.; Cai, Y.; Kwok, R. T.;
Lam, J. W. Y.; Gao, X.; Sun, P.; Phillips, D. L.; Ding, D.; Tang B. Z. Nat. Commun. 2019, 10, 768.

(33) Popa-Wagner, A.; Mitran, S.; Sivanesan, S.; Chang, E.; Buga, A.-M. Oxid. Med. Cell. Longev. 2013, 2013, 963520.

(34) Angelova, P. R.; Abramov, A. Y. FEBS Lett. 2018, 592, 692-702.

(35) Yanai, T.; Tew, D. P.; Handy, N. C. Chem. Phys. Lett. 2004, 393, 51-57.

(36) Adamo, C.; Jacquemin, D. Chem. Soc. Rev. 2013, 42, 845-856.

(37) Pugsley, H. R. Methods 2017, 112, 147-156. (38) Zhao, L.; Zhu, Y.; Wang, D.; Chen, M.; Gao, P.; Xiao, W.; Rao, G.; Wang, X.; Jin, H.; Xu, L. Autophagy 2010, 6, 386-394.

(39) Goldman, S. Nat. Biotechnol. 2005, 23, 862871.

(40) June, C. H. J. Clin. Investig. 2007, 117, 14661476. 

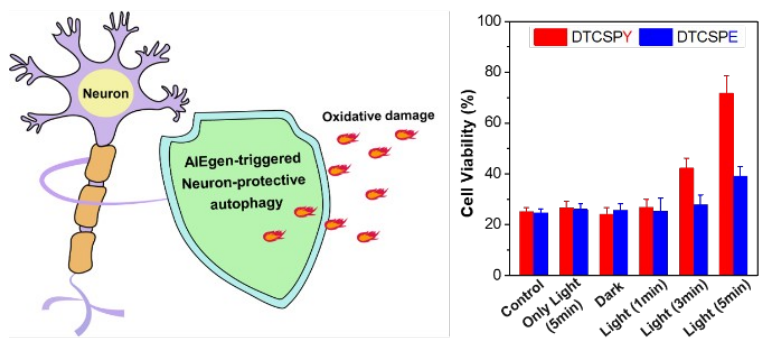